\title{
Low National Breast and Cervical Cancer-Screening Rates in American Indian and Alaska Native Women With Diabetes
}

\author{
Jennifer Giroux, $M D$, Thomas $K$. Welty, $M D, M P H$, Floyd $K$. Oliver, $P A$, \\ Judith S. Kaur, MD, Gary Leonardson, $P b D$, and Nathaniel Cobb, $M D$
}

Background: The cervical cancer mortality rate for American Indian and Alaska Native women is twice that of all races in the United States. To date the only published national breast and cervical cancerscreening rates for American Indian and Alaska Native women are based on self-reported data. When the Indian Health Service (IHS) conducts an annual audit on patients with diabetes, it includes cancer screening. This observational study presents national breast and cervical cancer-screening rates for American Indian and Alaska Native women with diabetes.

Metbods: Cancer-screening rates were extracted from the 1995 diabetic audit for the 12 IHS areas. These rates were compared with rates for women without diabetes of the same age, 50 to 69 years, by chart review, at four IHS hospitals in the Aberdeen IHS area.

Results: Screening rates for women with diabetes in the 12 areas varied: mammogram (ever) 35\% to $\mathbf{7 8 \%}$; clinical breast examination (last year) $28 \%$ to $70 \%$, and Papanicolaou smear (last year) $26 \%$ to $69 \%$. The Aberdeen IHS area women with diabetes had $51 \%$ more clinic visits per year than women without diabetes, but the groups had similar screening rates.

Conclusion: Cancer-screening rates for American Indian and Alaska Native women vary by region. In the Aberdeen IHS area, women with diabetes had more visits (missed opportunities) but similar screening rates as women without diabetes. The diabetic audit could be used to monitor national IHS cancerscreening trends for women with diabetes and in the Aberdeen IHS area for all women aged 50 to 69 years. (J Am Board Fam Pract 2000;13:239-45.)

The Indian Health Service (IHS) is responsible for providing health services to federally recognized tribes. The IHS is composed of 12 regional administrative units called Area Offices (Figure 1). Health services are offered to eligible American Indians and Alaska Natives through tribal or IHS-admin-

Submitted, revised 31 December 1999.

From the Epidemiology Program OG, TKW, FKO, JSK GL, NC), Aberdeen Indian Health Service Area, Rapid City, SD. Address reprint requests to Jennifer Giroux, MD, Center of American Indian and Minority Health, University Office Plaza, Suite 100, 211 University Ave SE, Minneapolis, MN 55414.

This research was supported in part by a National Cancer Institute grant (5 R01 CA 64321-01 \& 02) (Thomas K. Welty, MD, MPH, Principal Investigator, Jennifer Giroux, MD, Grant Coordinator). Dr. Giroux authored this article while an Epidemic Intelligence Service Officer, Centers for Disease Control and Prevention/Indian Health Service, under the supervision of Nathaniel Cobb, MD, IHS National Epidemiology Program. Judith Kaur, MD, was supported by a Bush Fellowship to study cancer prevention and control in American women while on staff at the Division of Medical Oncology, Mayo Clinic, Rochester, Minn.

The opinions expressed in this report are those of the authors and do not necessarily reflect those of the Indian Health Service. istered clinics or hospitals. In fiscal year 1997, there were 1.43 million American Indians and Alaska Natives who used IHS services. ${ }^{1}$

Both type 2 diabetes and cancer are serious chronic health problems for American Indians and Alaska Natives. ${ }^{2-4}$ Approximately one third of American Indians aged 45 years or older have diabetes. The Strong Heart Study documented that among American Indian women aged 45 to 74 years, $43 \%$ in the Dakotas, $41 \%$ in Oklahoma, and $71 \%$ in Arizona had type 2 diabetes; ${ }^{3}$ and the prevalence rates increased $11 \%$ in the Dakotas, $9 \%$ in Oklahoma, and 7\% in Arizona in 4 years (personal correspondence, Thomas K. Welty, Strong Heart Study, PI). Persons with diabetes represent an increasing proportion of the American Indian and Alaska Native population.

Cancer is second only to heart disease in causes of American Indian and Alaska Native deaths. ${ }^{5}$ In the 12 administrative IHS areas, mortality rates vary considerably for breast cancer (4.2-26.3/ $100,000)$ and cervical cancer(2.7-15.6/100,000). ${ }^{2}$ 


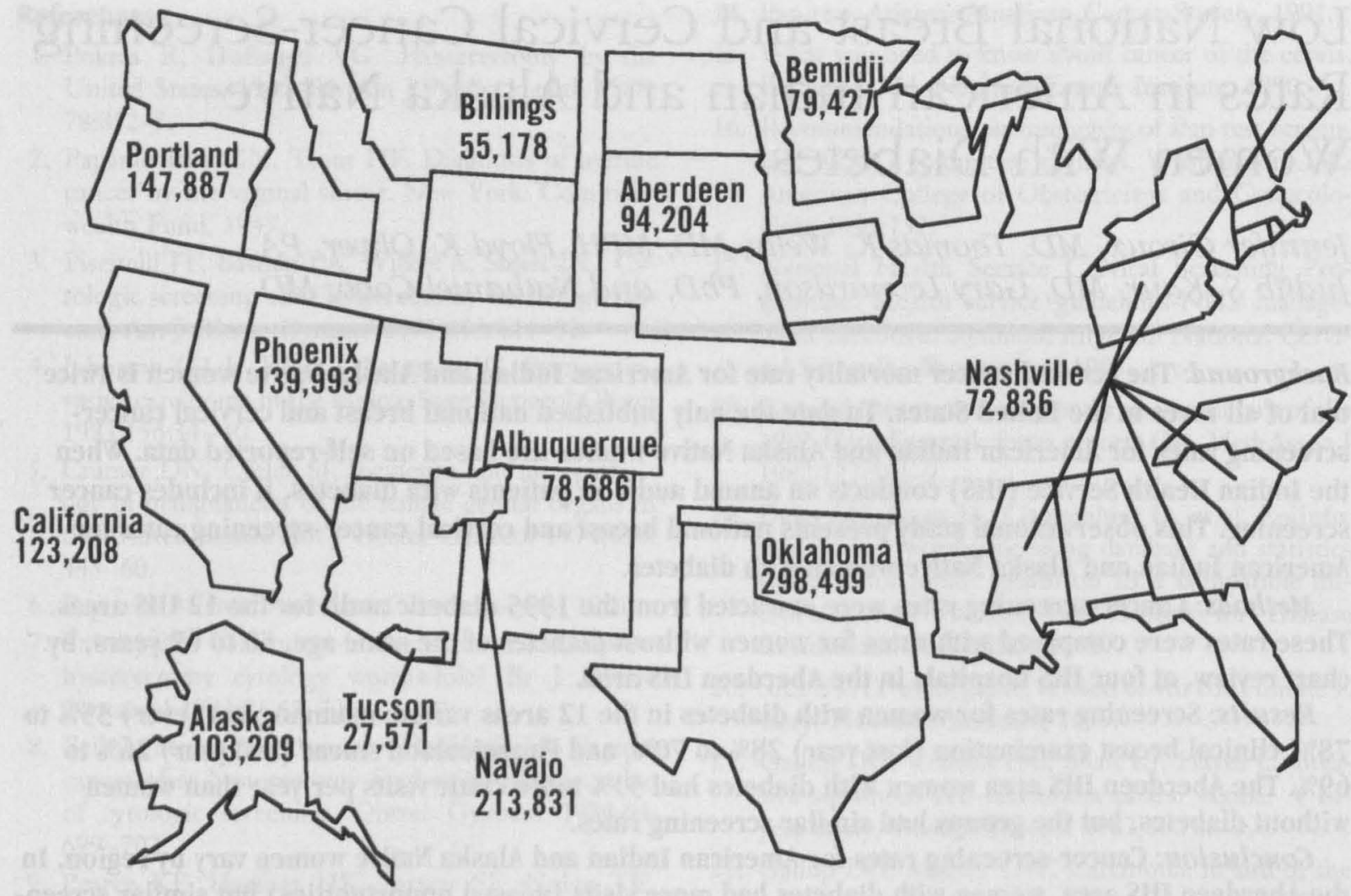

Figure 1. Indian Health Service population by area, fiscal year 1997.

Most IHS areas, except Aberdeen and Billings, are below the year 2000 goal of a rate of 25.2 per 100,000 for breast cancer mortality; ${ }^{6}$ but American Indian and Alaska Native women have the poorest 5 -year survival rate from breast cancer of any racial group. ${ }^{2}$ The IHS does not have its own guidelines for breast cancer screening but follows the $\mathrm{Na}$ tional Institutes of Health consensus guidelines.

All IHS areas have uterine cervical cancer mortality rates higher than the year 2000 goal of 1.5 per $100,000 .^{7}$ In the Aberdeen IHS area, the cervical cancer mortality rate is five times that of all races in the United States (15.6 vs 3.0). The IHS recommends cervical cancer screening every year from age 18 years or from the onset of sexual activity. To date, the only published national breast and cervical cancer-screening rates for American Indian and Alaska Native women is based on self-reported behavioral risk factor surveillance data.

The IHS Diabetes Control Program conducts the Diabetes Audit, a quality-improvement audit, for patients with diabetes once a year according to nationally approved standards that include interventions for which preventive and therapeutic benefits have been scientifically determined. ${ }^{8,9}$ These
Diabetes Audit standards include documentation of cancer screening (Papanicolaou smear, mammogram, and clinical breast examination [CBE], and rectal examination). For the Aberdeen IHS area, we wanted to determine whether breast and cervical cancer-screening rates for women with diabetes were representative of women without diabetes. Doing so would allow us to assess whether it was feasible to use the Diabetes Audit to monitor longitudinal cancer-screening trends for all American Indian women aged 50 years or older in the Aberdeen IHS area.

We hypothesized that patients with diabetes are more likely to obtain cancer screening than patients without diabetes because national standards of care for diabetic patients include cancer screening, and diabetic patients are seen more frequently for medical care.

\section{Methods}

The IHS Diabetes Program audits medical records from a representative sample of patients with type 2 diabetes in all IHS facilities. At each service unit, the number of charts audited is derived from sam- 
ple size calculations that are based on the total number of patients in the diabetic registry. This sample size allows for at least a $90 \%$ confidence that rates found are within $10 \%$ of the true population rate. Cancer-screening standards have been audited in a uniform way at all IHS facilities during 1988 to 1997 . National screening rates for breast and cervical cancer screening were extracted from the 1995 audit.

The IHS patient care data system includes a computerized record for each outpatient visit with the purpose of visit coded by International Classification of Diseases-9-Clinical Modification (ICD9-CM) criteria. ${ }^{10}$ At four IHS hospitals in the Aberdeen IHS area, two printouts were obtained, one for women aged 50 to 69 years seen for diabetes mellitus (ICD-9-CM code 250) $(n=664)$, and the other for all women aged 50 to 69 years $(n=1880)$. Both groups received some type of health care from the service unit in the last 12 months.

We randomly selected 100 medical charts from each of these two groups at each of the four hospitals. Fewer charts were audited at one hospital (hospital A) because of person-power and time constraints. We validated the abstraction process by duplicating abstraction efforts to assure audits were done in a uniform manner.
Women whose residence was not in the service delivery area, who died, or who had not been seen by a primary care provider at the IHS facility in the last year were excluded from this study. This exclusion allowed us to assess missed opportunities for breast and cervical cancer screening. At several service units adequate person-power and time allowed us to audit additional medical charts to replace the audits that were excluded.

The number of randomly selected charts from each group ranged from 49 to 100 at the four sites. Overall, medical charts were audited for approximately $50 \%$ of the patients with diabetes and $17 \%$ of patients without diabetes at these four hospitals. The service population at these four hospitals represented $37 \%$ of entire service population in the Aberdeen IHS area. An additional five hospitals and seven health centers in the Aberdeen IHS area were not included in this study.

We used an abstraction tool that contained the Diabetes Audit and some additional information, including the number of outpatient contacts with primary care providers in the last year.

Statistical significance of differences in screening rates were determined by chi squares. $P<.05$ was considered to be statistically significant.

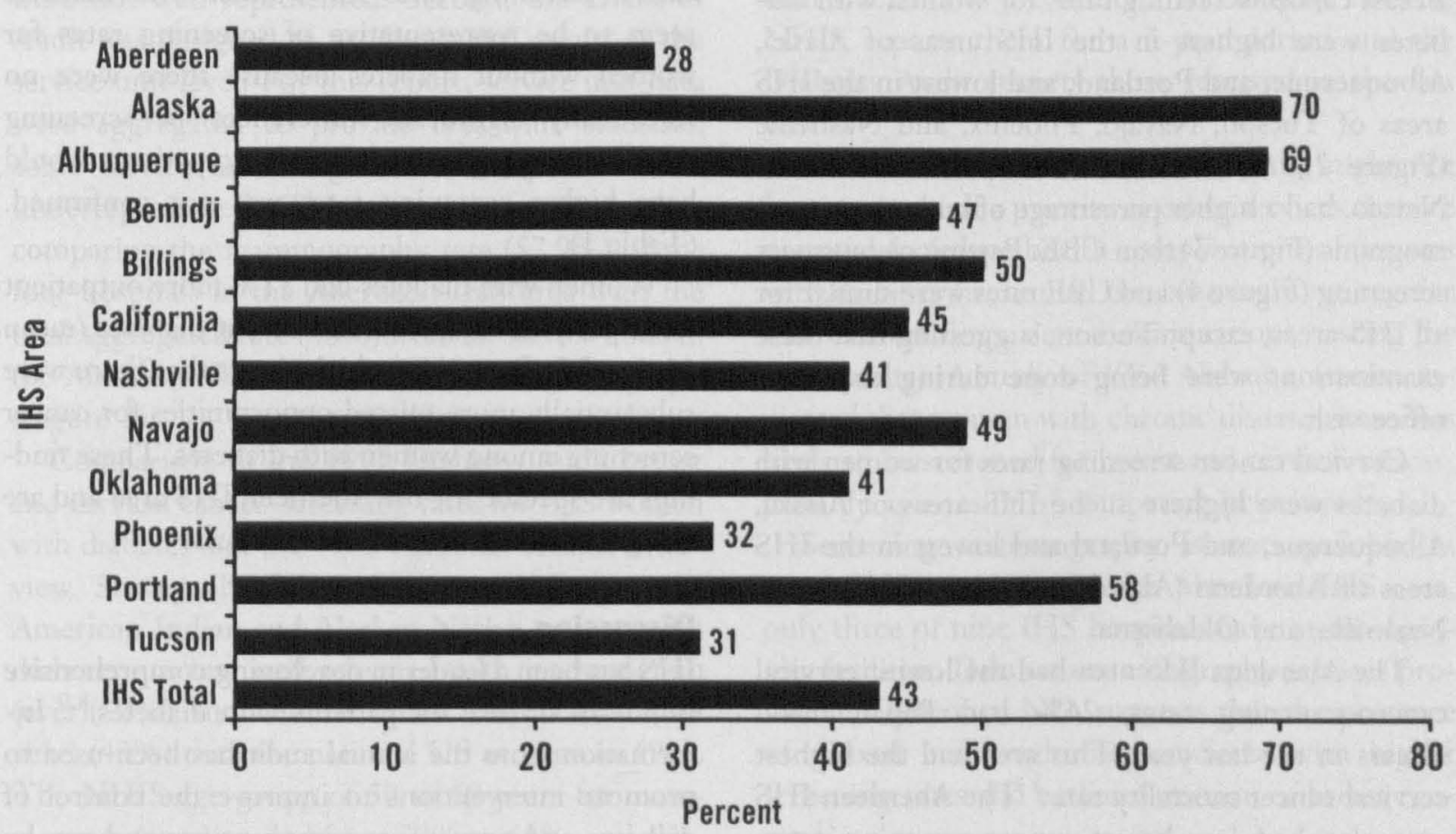

Figure 2. Percentage of American Indian and Alaskan Native with diabetes receiving clinical breast examination in the last year. Data from the 1995 Indian Health Service Audit. 


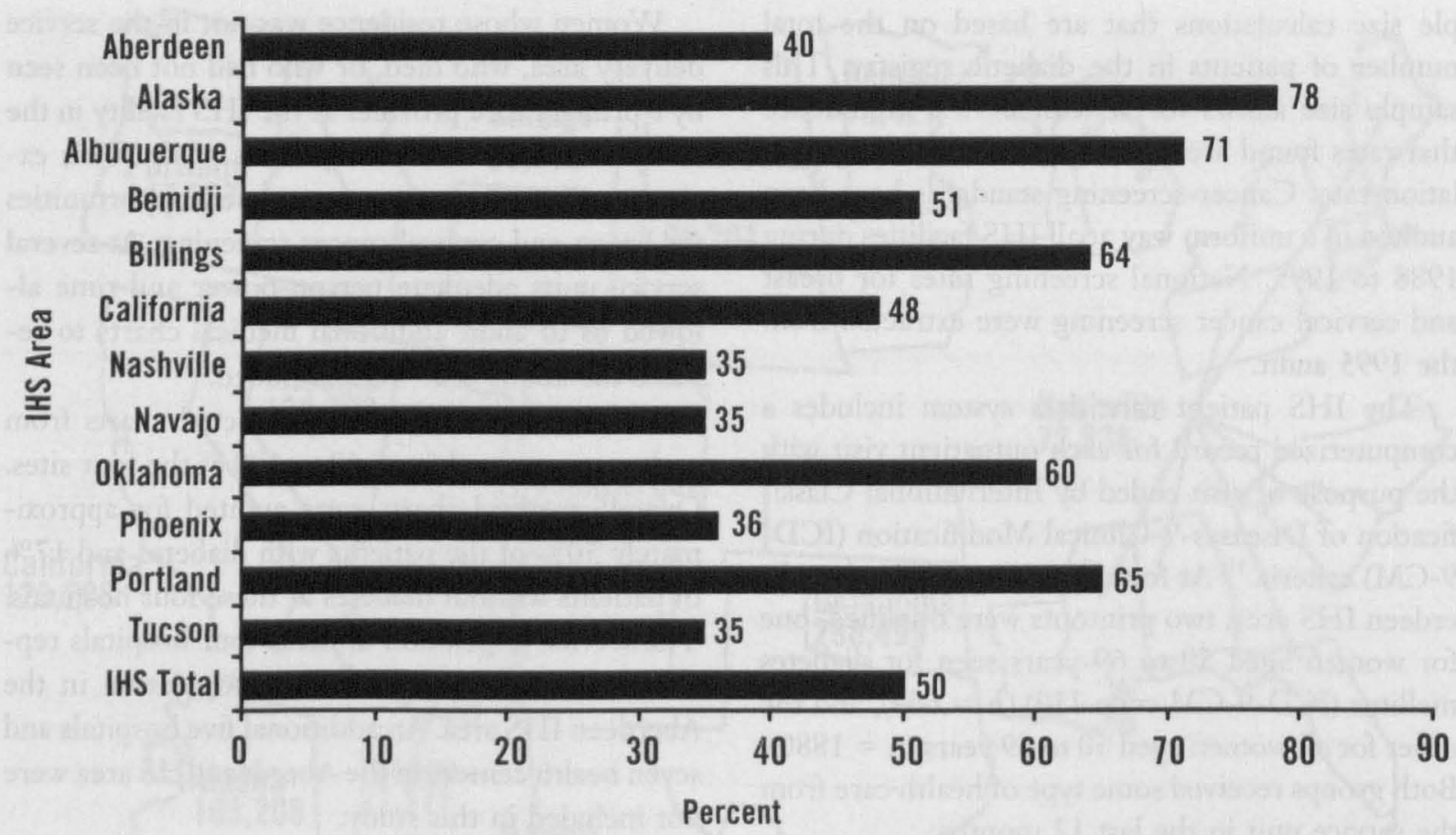

Figure 3. Percentage of American Indian and Alaska Native women with diabetes receiving mammogram ever. Data from the 1995 Indian Health Service Audit.

\section{Results}

The 1995 Diabetes Audit for patients with diabetes documented that breast and cervical cancer-screening rates varied throughout the 12 IHS areas. Breast cancer-screening rates for women with diabetes were highest in the IHS areas of Alaska, Albuquerque, and Portland, and lowest in the IHS areas of Tucson, Navajo, Phoenix, and Nashville (Figure 2). All IHS areas, except Nashville and Navajo, had a higher percentage of screening mammograms (Figure 3) than CBE. Papanicolaou smear screening (Figure 4) and CBE rates were similar for all IHS areas, except Tucson, suggesting that these examinations were being done during the same office visit.

Cervical cancer-screening rates for women with diabetes were highest in the IHS areas of Alaska, Albuquerque, and Portland and lowest in the IHS areas of Aberdeen (Aberdeen IHS area), Phoenix, Nashville, and Oklahoma.

The Aberdeen IHS area had the lowest cervical cancer-screening rates; $26 \%$ had Papanicolaou smears in the last year. This area had the highest cervical cancer mortality rate. ${ }^{1}$ The Aberdeen IHS area also had low breast cancer-screening rates: $40 \%$ ever had mammogram and $28 \%$ had a CBE in the last year. Alaska IHS area had the highest breast and cervical cancer-screening rates: $78 \%$ ever had mammogram, $70 \%$ had a CBE in the last year, and $69 \%$ had Papanicolaou smears in the last year.

In the Aberdeen IHS area, breast and cervical cancer-screening rates for women with diabetes seem to be representative of screening rates for women without diabetes because there were no statistically significant differences in screening rates. Our hypothesis that diabetic patients would have higher screening rates was not confirmed. (Table 1)

Women with diabetes had $51 \%$ more outpatient visits per year than women without diabetes (mean 11.6 vs $7.7, P=.001$ ), which shows that there were substantially more missed opportunities for cancer screening among women with diabetes. These findings are specific for the Aberdeen IHS area and are not generalizable to other IHS areas.

\section{Discussion}

IHS has been a leader in developing comprehensive standards of care for patients with diabetes. ${ }^{8,9} \mathrm{In}$ formation from the annual audit has been used to promote interventions to improve the control of diabetes and prevent its complications and can be used to monitor various health indicators in the diabetic population served by IHS. 


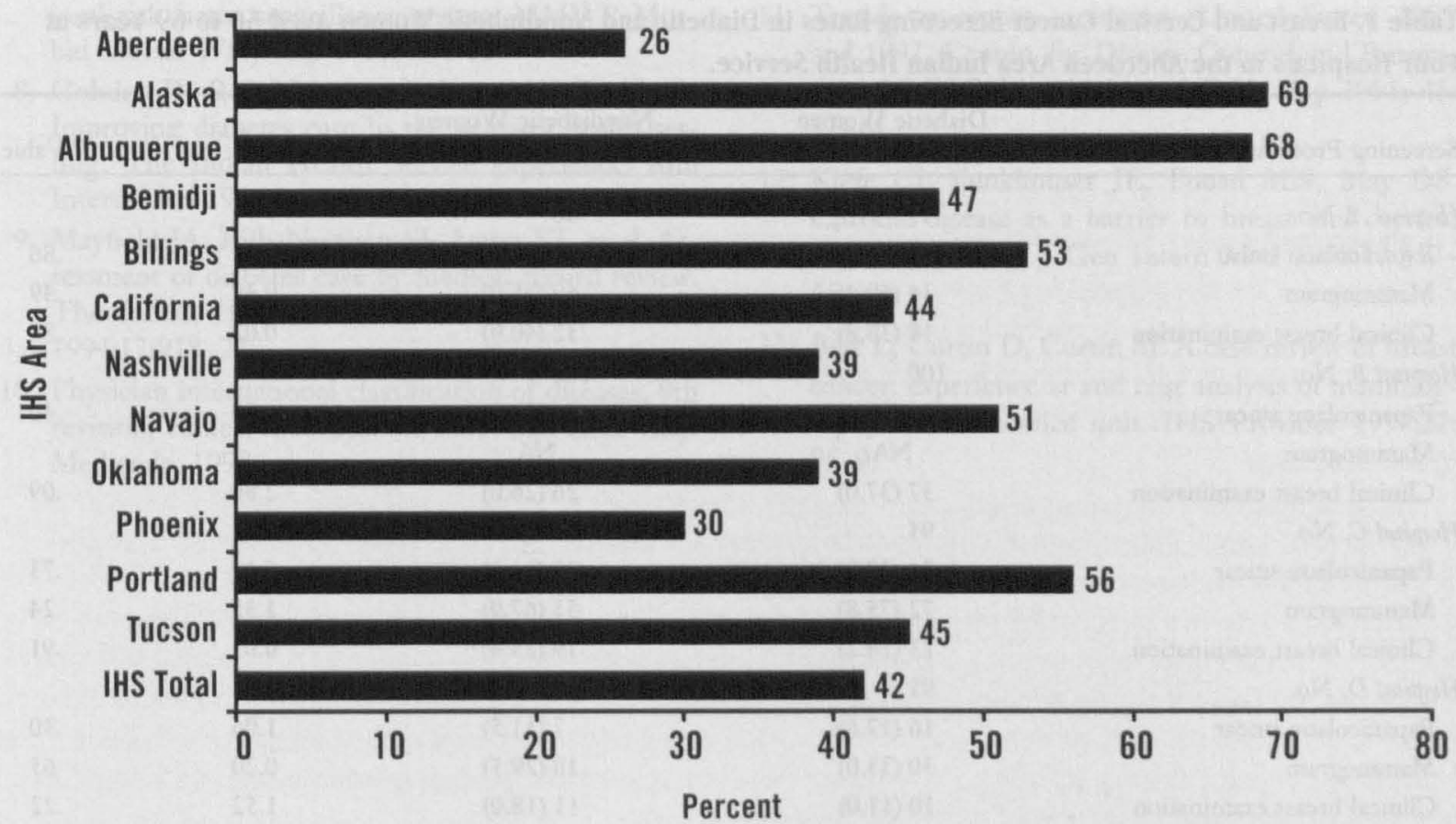

Figure 4. Percentage of American Indian and Alaskan Native women with diabetes receiving a Papanicolaou smear in the last year. Data from the 1995 Indian Health Service Audit.

Several limitations of the Diabetes Audit exist. First, participation is voluntary at the service unit level. Although most IHS facilities chose to participate, two IHS Areas, California and Nashville, were not well represented. Second, the Diabetes Audit was designed to produce valid results at the service unit level. For this report, service unit data were aggregated to provide area-wide statistics; some service units might be overrepresented or underrepresented. This situation is seen when comparing the mammography rate $(57.9 \%)$ of the four hospitals in the Aberdeen IHS area with the total aggregated rate ( $40 \%$ ) from all service units in the Aberdeen IHS area in the mammogram graph (Figure 3).

Comparison of the 1995 Diabetes Audit breast and cervical cancer-screening rates for IHS women with diabetes and the 1992 National Health Interview Survey (NHIS) indicates overall in 1995 American Indian and Alaskan Native women with diabetes are less well screened with mammogram (ever) $(50 \%)$ and clinical breast examination (last year) $(43 \%)$ than the national US average in 1992. The NHIS age-group of 50 to 69 years for mammogram (ever) ranged from $59 \%$ to $64 \%$, and clinical breast examination (last year) ranged from $49 \%$ to $55 \%$. Because we excluded from our study women who had not been to a primary care provider in the last year, and the NHIS did not, our rates overestimate breast and cervical cancerscreening rates compared with NHIS. Papanicolaou smear rates are difficult to compare because NHIS reports them for a 3-year period and the Diabetes Audit reports those received in the last year. ${ }^{11}$

Breast and cervical cancer screening might not be given as high a priority as other services that are essential to reduce health complications of persons with diabetes (ie, annual eye examinations, regular foot examinations, dental examinations, testing for neuropathy). A study of 1764 Alabama women indicated that women with chronic diseases were less likely to be screened for breast and cervical cancer, possibly as a result of competing priorities. ${ }^{12}$

Screening mammography is not available in many IHS locations. In the Aberdeen IHS area, only three of nine IHS hospitals have mammography facilities. Data on mammography services provided at Belcourt, ND, suggests that the presence of a fixed unit might be associated with higher screening rates and earlier detection of breast cancer. $^{13}$

In summary, national breast and cervical cancerscreening rates vary by region. When compared 
Table 1. Breast and Cervical Cancer Screening Rates in Diabetic and Nondiabetic Women Aged 50 to 69 Years at Four Hospitals in the Aberdeen Area Indian Health Service.

\begin{tabular}{lcccc}
\hline Screening Procedure & $\begin{array}{c}\text { Diabetic Women } \\
\text { No. (\%) }\end{array}$ & $\begin{array}{c}\text { Nondiabetic Women } \\
\text { No. }(\%)\end{array}$ & $\chi^{2}$ & $P$ Value \\
\hline Hospital $A$, No. & 49 & 80 & & \\
Papanicolaou smear & $17(34.7)$ & $29(36.3)$ & 0.03 & .86 \\
Mammogram & $34(69.4)$ & $60(75.0)$ & 0.48 & .49 \\
Clinical breast examination & $19(38.8)$ & $32(40.0)$ & 0.02 & .89 \\
Hospital $B$, No. & 100 & 100 & & \\
Papanicolaou smear & $21(21.0)$ & $26(26.0)$ & 0.70 & .40 \\
Mammogram & NA & NA & & \\
Clinical breast examination & $37(37.0)$ & $26(26.0)$ & 2.80 & .09 \\
Hospital C, No. & 95 & 81 & & \\
Papanicolaou smear & $34(35.8)$ & $27(33.3)$ & 0.12 & .73 \\
Mammogram & $72(75.8)$ & $55(67.9)$ & 1.35 & .24 \\
Clinical breast examination & $23(24.2)$ & $19(23.4)$ & 0.01 & .91 \\
Hospital D, No. & 91 & 61 & & \\
Papanicolaou smear & $16(17.6)$ & $7(11.5)$ & 1.06 & .30 \\
Mammogram & $30(33.0)$ & $18(29.5)$ & 0.20 & .65 \\
Clinical breast examination & $10(11.0)$ & $11(18.0)$ & 1.52 & .22 \\
Total, No. & 335 & 322 & & \\
Papanicolaou smear & $88(26.3)$ & $38(27.6)$ & 0.16 & .69 \\
Mammogram & $136(57.9)$ & $133(59.9)$ & 0.20 & .66 \\
Clinical breast examination & $89(26.6)$ & $88(27.3)$ & 0.05 & .83 \\
\hline
\end{tabular}

with Aberdeen IHS area women without diabetes, women with diabetes had more clinic visits but similar breast and cervical cancer-screening rates; therefore, diabetic women had more missed opportunities for preventive cancer screening than nondiabetic women.

Early diagnosis through periodic screening is key to reducing breast and cervical cancer mortality rates. In areas where screening rates are low, the barriers must be assessed and an effort made to implement innovative approaches to increase the cancer-screening rates.

This report was made possible with the assistance of the staff at IHS facilities throughout the country who are dedicated to improving the quality of care for their patients. We appreciate the IHS Diabetes Programs that coordinate and conduct chart audits; medical records staff at the IHS facilities in Belcourt, Eagle Butte, Rapid City and Rosebud. Aruna Das, MD, MPH, Daniel Curtin, MD, Michelle Curtin, MEd, Lori Dablemont, MD, Saeed Mahmoodian, MD, and Susan Stackelhouse, MD, helped abstract records at the hospitals in the Aberdeen IHS area. Tribal approval and support for this research and publication made this report possible.

\section{References}

1. Cobb N, Paisano RE. Cancer mortality among American Indians and Alaska Natives in the United
States: regional differences in Indian health 1989 1993. Rockville, Md: Indian Health Service, 1997. (IHS publication no. 97-615-23.)

2. Burhansstipanov L, Dresser CM. Documentation of the cancer research needs of American Indians and Alaska Natives. Native American monograph no. 1. Bethesda, Md: National Institutes of Health, 1993; D-2. (NIH publication no. 93-3603.)

3. Lee ET, Howard BV, Savage PJ, et al. Diabetes and impaired glucose tolerance in three American Indian populations aged $45-74$ years. The Strong Heart Study. Diabetes Care 1995;18:599-610.

4. Welty TK, Zephier N, Schweigman Kurt, Blake B, Leonardson G. Cancer risk factors in three Sioux tribes. Use of the Indian-specific health risk appraisal for data collection and analysis. Alaska Med 1993;35: 265-72.

5. Trends in Indian health. Washington, DC: US Department of Health and Human Services, Public Health Service, Indian Health Service, Office of Planning, Evaluation, and Legislation, Division of Program Statistics, 1996

6. Healthy people 2000 , national promotion and disease prevention objectives. Hyattsville Md: US Department of Health and Human Services, Public Health Service, 1990. (DHHS publication no. [PHS] 91-50213.)

7. Trends in self-reported use of mammograms (19871997) and Papanicolaou tests (1991-1997): behav- 
ioral risk factor surveillance system. MMWR Morbid Mortal Wkly Rep 1999;48:1-22.

8. Gohdes D, Rith-Najarian S, Acton K, Shields R. Improving diabetes care in the primary health setting. The Indian Health Service experience. Ann Intern Med 1996;124(1 Pt 2):149-52.

9. Mayfield JA, Rith-Najarian SJ, Acton KJ, et al. Assessment of diabetes care by medical record review. The Indian Health Service model. Diabetes Care 1994;17:918-23.

10. Physician international classification of diseases, 9 th revision, clinical modification, ed 5. Salt Lake City: Medicode, 1998.
11. Trends in cancer screening-United States. 1987 and 1992. Centers for Disease Control and Prevention. MMWR Morbid Mortal Wkly Rep 1995; 45: 57-61.

12. Kiefe CI, Funkhouser JE, Fouad MN, May DS. Chronic disease as a barrier to breast and cervical cancer screening. J Gen Intern Med 1998;13:35765.

13. Best $L$, Curtin D, Curtin $M$. A case review of breast cancer: experience at and cost analysis of mammography at one service unit. IHS Provider 1997;22: 95-9. 